\title{
Revival of Renal Denervation Therapy for Hypertension: Real Hope or just a Dream?
}

\author{
${ }^{1}$ Venkatesh K Raman, ${ }^{2}$ Vasilios Papademetriou
}

\section{ABSTRACT}

The sympathetic nervous system (SNS) has long been recognized as important to the initiation and maintenance of hypertension. From groundbreaking experimental physiology in the 1850 s to single nerve fiber studies more than one 100 years later, the growing body of preclinical evidence supports this framework and has informed attempts at therapeutic clinical intervention. Prior to the availability of long-term oral antihypertensive therapy in the mid-1950s, operative approaches to SNS interruption were of variable efficacy and sometimes limiting side effects. Surgery was then abandoned as a panoply of drugs were introduced and shown to be effective in reducing blood pressure and, more importantly, decreasing major cardiovascular events. Despite the availability of dozens of agents, a small but significant percentage of patients do not achieve target pressures on multiple medications and are characterized as resistant or refractory. Nonsurgical, catheter-delivered radiofrequency ablation (RFA) to achieve renal SNS denervation has emerged as an option in these patients. Early clinical studies suggested marked reductions in office-based blood pressures. Results of the pivotal, sham-controlled SYMPLICITY HTN-3 study, however, were disappointing and did not show a significant difference in blood pressure reduction between treated and control groups. Post hoc analyses suggest that incomplete denervation was an important contributor to the much smaller blood pressure effect compared with earlier trials. Additional clinical anatomic and preclinical studies have greatly elucidated the distribution of sympathetic nerves in the renal periarterial space and provided insight into broad principles that might successfully move the field forward. A number of newer treatment platforms that include multielectrode RFA, focused high-energy ultrasound, and local periarterial delivery of neurolytics seem to justify the cautious enthusiasm that renal denervation (RDN) will eventually fulfill its promise in the treatment of resistant hypertension and perhaps other syndromes characterized by sympathetic overactivity.

Keywords: Ambulatory blood pressure monitoring, Norepinephrine, Radiofrequency ablation, Renal denervation, Renal sympathetic nervous activity, Sympathetic nervous system.

How to cite this article: Raman VK, Papademetriou V. Revival of Renal Denervation Therapy for Hypertension: Real Hope or just a Dream? Hypertens J 2016;2(3):160-168.

${ }^{1}$ Assistant Professor, ${ }^{2}$ Director and Professor

${ }^{1}$ Department of Medicine, MedStar Heart and Vascular Institute MedStar Georgetown University Hospital, Washington, District of Columbia, USA

${ }^{2}$ Department of Hypertension and Interventional Cardiology Veteran Affairs Medical Center and Georgetown University Washington, District of Columbia, USA

Corresponding Author: Vasilios Papademetriou, Director and Professor, Department of Hypertension and Interventional Cardiology, Veteran Affairs Medical Center and Georgetown University Washington, District of Columbia, USA, e-mail: vpapademetriou@va.gov
Source of support: Nil

Conflict of interest: None

\section{INTRODUCTION}

The influence of renal sympathetic nervous activity (RSNA) on renal function and, consequently, on water and sodium homeostasis has long been known (Fig. 1). In the mid-nineteenth century, the French physiologist Claude Bernard reported that unilateral section of the splanchnic nerve in the anesthetized dog resulted in marked diuresis, while electrical stimulation of the cut nerve end had the opposite effect. ${ }^{1}$ Bradford subsequently showed in dogs that dorsal and splanchnic nerve stimulation led to changes in blood pressure, with the direction dependent upon the anatomic location of the applied stimulus as well as pulse frequency. ${ }^{2}$ Elucidation of the anatomic extent and distribution of nerve input would follow over the next several decades, along with an understanding of the kidney as originator and recipient of sympathetic input. Afferent input from mechanoreceptors and chemoreceptors in the renal pelvis sends signals to the central nervous system that results in sympathetic overactivity in response to injury and changes in urine composition as well as to increases in renal pelvic pressure. ${ }^{3}$ Efferent fibers from the brain reach the kidneys through the spinal cord and 2nd sympathetic ganglion, running within the renal artery adventitia and innervating arterioles, tubular epithelial cells, and the juxtaglomerular apparatus. Activity in these fibers leads to increased renin secretion, increased tubular sodium reabsorption, and renal vasoconstriction with a decrease in renal blood flow (RBF) (Fig. 1).

\section{Sympathetic Activity and Denervation in Preclinical Models}

The contribution of the renal sympathetic nerve activity in the development and maintenance of hypertension is complex, multifaceted, and variable, yet well established. The relationship between water-sodium balance and arterial pressure has led to the idea of a central role for the sympathetic nervous system (SNS). Abnormal renal function may be driven by excessive RSNA via release of vasoconstrictors, increased vascular resistance, and decreased RBF. The resulting altered pressure natriuresis 


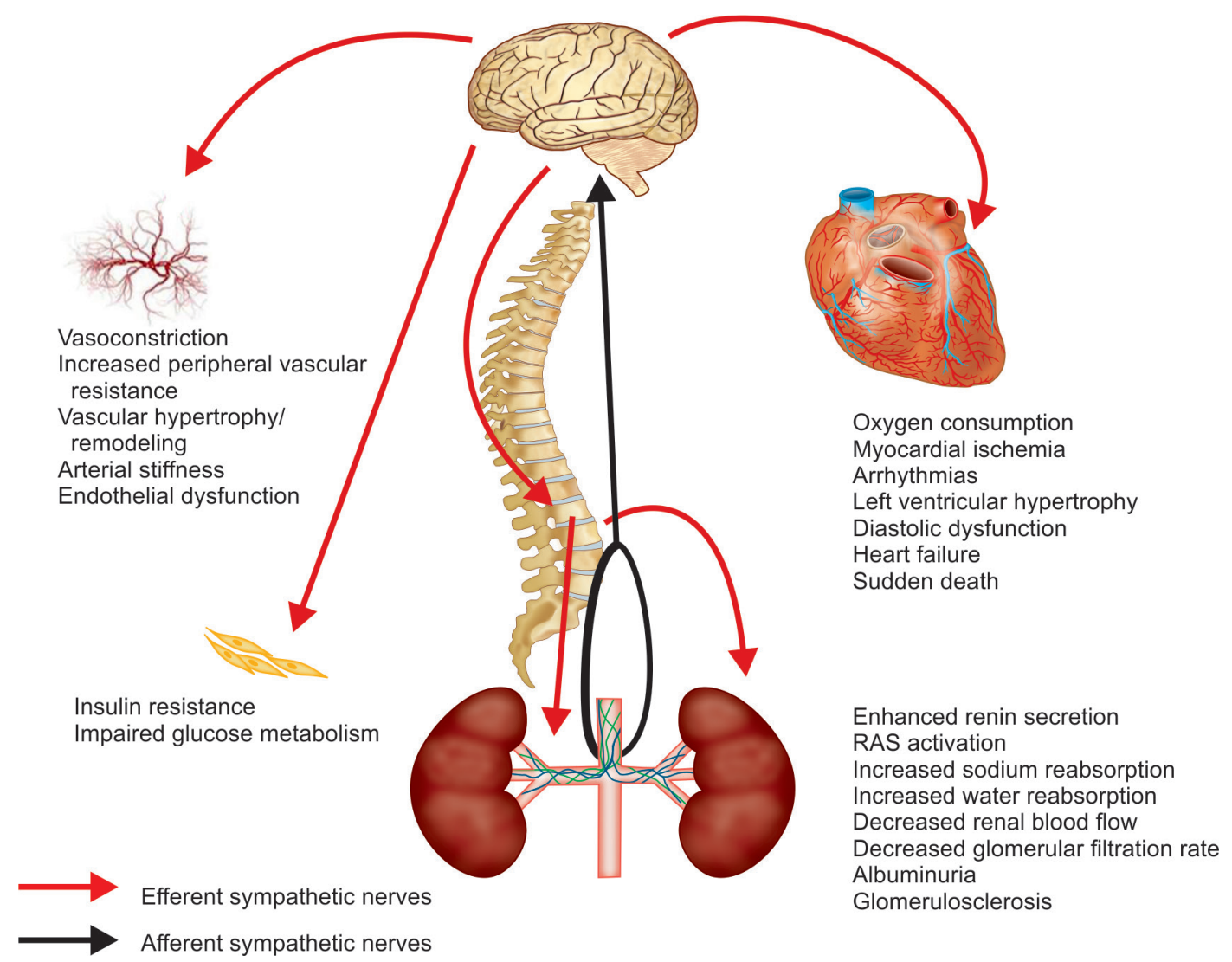

Fig. 1: Schematic presentation of sympathetic nervous system and influence of efferent and afferent sympathetic nerve fibers

and diuresis curves must then be overcome by permissive arterial hypertension to restore sodium balance. Chronic stimulation of the renal artery nerves was observed in a dog model to produce hypertension for the duration of its application. ${ }^{4}$ Electrical stimulation of afferent sympathetic fibers in a rat model reduced blood pressure in a dose-dependent manner, the effect of which was abrogated following spinal transection. ${ }^{5}$ Malpas and Evans ${ }^{6}$ demonstrated that activation of renal efferent nerves resulted in renal vasoconstriction. The contribution of sympathetic activity to experimental hypertension was convincingly shown by Thorén ${ }^{7}$ in a demonstration of increased single-fiber RSNA in spontaneously hypertensive rats compared with normotensive Wistar-Kyoto rats.

With this background establishing the connection between RSNA and experimental hypertension, a number of investigators explored the effect of sympathetic interruption on initiation and maintenance of these states. In a series of experiments with a renal injury-induced hypertension model in rats, Campese and Kogosov ${ }^{8}$ prevented the development of hypertension through dorsal rhizotomy, selectively interrupting renal afferent sympathetic input, and even slowed the progression of renal disease with less severe glomerulosclerosis. ${ }^{9}$ In deoxycorticosterone acetate (DOCA)-treated miniature swine with established hypertension, surgical renal denervation (RDN) led to a marked decrease in blood pressure without natriuresis. ${ }^{10}$ In another set of experiments, young rats underwent single nephrectomy and denervation of the contralateral kidney one week before DOCA-salt treatment. Blood pressure was significantly higher in the sham-operated group, increasing by day 5 and reaching a plateau by 3 weeks. In contrast, DOCAsalt administration in the denervated group did not lead to a blood pressure rise during the 1st 2 weeks. By the 3rd week, however, investigators observed a rapid rise in blood pressure and a fall in fractional urinary sodium excretion to the level of the sham-operated controls. They noted a concomitant threefold increase in renal norepinephrine (NEPI) content, consistent with reinnervation. ${ }^{11}$ Huang et al, ${ }^{12}$ using a hyperinsulinemia-induced hypertension model in rats, showed that renal nerve denervation could both prevent development of hypertension and normalize blood pressures within 2 weeks once established, demonstrating the obligatory role of RSNA in this model. In fact, the effectiveness of RDN in a variety of models of hypertension - genetic, renal injury, pharmacologic - supports an important pathophysiologic role for renal sympathetic activity.

\section{Early Experience with Clinical Denervation}

Despite the promising results in multiple experimental models, early results in humans were at times conflicting. 
Renal sympathetic nerve resection was done surgically and was staged for safety reasons. Early surgical sympathectomy in the 1920s was followed by numerous reports from multiple centers in the next two decades. ${ }^{13,14}$ Renal decapsulation, also considered a form of sympathectomy by disruption of fibers between the capsule and cortex, was performed in patients with unexplained hematuria and perinephritis. A significant but only temporary reduction in blood pressure was noted in this series of 85 patients. ${ }^{15}$ Page and Heuer presented the 1st report of bilateral RDN to treat severe hypertension in a 25-year-old woman. The patient had blood pressures over 200/100 mm Hg and underwent staged operative denervation separated by 1 month. Although the procedure was well tolerated and without "ill effects" on renal function, there was no significant reduction in blood pressure. ${ }^{16}$ A small number of additional patients also underwent this treatment with short-term blood pressure reduction but no long-term effects. Consequently, a more dramatic approach was taken by Freyberg and Peet, ${ }^{13}$ who performed splanchnicectomy in a series of 48 patients with severe hypertension [systolic blood pressure (SBP) >200 mm Hg]. His team noted varied effects, "from complete relief of hypertension, in some, to complete failure in others...."

They found no decrement in kidney function measured by concentrating ability and urea clearance, and they noted that favorable changes in renal function followed such changes in blood pressure. These human data clearly demonstrate that the effect of even complete sympathetic denervation on blood pressure is variable. It may be unrealistic to expect complete and uniform response with less-than-complete sympathetic denervation.

For the next two decades, surgical splanchnicectomy became the treatment of choice for severe or malignant hypertension. Smithwick and Thompson ${ }^{17}$ reported 5-year results on 1,266 patients who had splanchnicectomy and 467 patients on medical therapy. Mortality in the surgical patients was reduced by two-thirds (19 vs $54 \%$ ). In patients with malignant hypertension, Peet $^{18}$ noted dramatic improvements in survival after operation compared with a reported cohort of patients treated medically. ${ }^{19}$ At the end of 5 years, only 1 out of 146 patients treated medically was still alive, while more than one-third of the surgical subjects were living. One case example with remarkable results involved a 22-year-old with SBP of $250 \mathrm{~mm} \mathrm{Hg}$ during pregnancy. She developed incapacitating headaches, suffered blurry vision, and had been confined to bed rest for 8 months. Two months after bilateral splanchnicectomy, the patient was asymptomatic with a blood pressure of 135/90, a result that persisted to the end of reported follow-up at 12 years. Despite these successes, the procedure was associated with significant side-effects, including limiting orthostatic hypotension and bowel and bladder dysfunction. With the availability of chlorothiazide in the 1950s, an effective and welltolerated therapy, surgical sympathectomy was largely abandoned. It is important to reiterate, however, that blood pressure reduction in about two-thirds of patients with severe or malignant hypertension was achieved with splanchnicectomy, a much more radical procedure than RDN. Results with the latter technique were much less impressive.

\section{Clinical Trials of Catheter-based Renal Denervation}

In the interim, as noted previously, there has been extensive study of the role of the SNS, particularly the renal sympathetic nerves, in hypertension. We have reviewed the compelling preclinical data supportive of a central role of RSNA in hypertension models. In the clinical realm, it has also been established that SNS overactivity is present in hypertension. Kjeldsen et $\mathrm{al}^{20}$ studied hypertensive-but-untreated and normotensive men over 50 years of age. They found moderately elevated plasma NEPI levels, representing sympathetic tone, in the group with established hypertension and postulated this as an important pathogenic factor. A group from St. James's University Hospital explored sympathetic activity across a spectrum of borderline hypertension, white coat hypertension, and various stages of essential hypertension (according to JNC-6). ${ }^{21}$ Elevated pulse frequency muscle sympathetic nerve activity was observed in borderline and early-stage hypertension, but to a lesser degree in stage 2 or 3 hypertension, compared with normotension and white coat hypertension. They concluded that sympathetic overactivity is likely to play an important role in the development of hypertension.

Advances in catheter-based technologies have coincided and allowed the concept of RDN to be clinically translated via a percutaneous approach. Radiofrequency ablation (RFA) has long been used in the treatment of cardiac rhythm abnormalities and tumors, effecting thermal destruction via deposition of energy from the electrode tip to target tissue. Accessory conduction bypass pathways, supraventricular tachycardia, and atrial fibrillation are among the intracardiac circuits that can be isolated, interrupted, or destroyed by RFA. Depth of tissue injury depends upon electrode tissue contact, power delivery, and tissue impedance. A series of experiments by Schauerte et al at the University of Oklahoma published in 1999 and 2000 opened up the possibility of a transvascular approach for RFA of extravascular targets. Placing an expandable basket catheter in the superior vena cava of dogs, they demonstrated slowing of the sinus rate by catheter stimulation of the vagal nerve, even 
during an isoproterenol infusion. ${ }^{22}$ These findings were extended to show that inducibility of atrial fibrillation could be abolished by RFA of parasympathetic pathways along the right pulmonary artery. ${ }^{23}$

This insight into perivascular nerve interruption provided the basis for the current catheter-based transvascular approach to RDN. Renal sympathetic nerves are known to course within the adventitia of the renal arteries and seemed to provide a readily accessible target for sympathoinhibitory treatment of hypertension. While there are more than a half-dozen CE mark-approved RDN platforms available in Europe and elsewhere throughout the world, none are available in the United States. Most of these devices use RFA to achieve denervation: Medtronic Symplicity (Dublin, Ireland), Boston Scientific Vessix V2 (Marlborough, MA, USA), Covidien OneShot (Dublin, Ireland), Terumo Iberis (Tokyo, Japan), St. Jude EnligHTN (St. Paul, MN, USA), Cordis Renlane (Fremont, CA, USA). ReCor's Paradise (Palo Alto, CA, USA) system uses high-intensity focused ultrasound for sympathetic interruption. Ablative Solutions' Peregrine System (Kalamazoo, MI, USA) delivers alcohol, a neurolytic, into the periarterial tissue to effect denervation. Medtronic's Symplicity system has the most extensive clinical experience and longest follow-up, and the important SYMPLICITY HTN trials will be discussed in detail below.

The early studies in humans, done with a singleelectrode catheter, were single-arm, uncontrolled, proofof-concept studies. Nevertheless, they were interpreted as demonstrating impressive benefits for blood pressure control. This spurred an unwarranted enthusiasm in the field, which was curtailed by the results of SYMPLICITY HTN-3. Our concerns and skepticism about the early clinical studies, but not the concept of RDN, have been previously published. ${ }^{24,25}$ We will briefly explore some of the issues related to the early studies and why the results of SYMPLICITY HTN-3 should not have been surprising.

The Symplicity system uses a single-electrode catheter to deliver lesions along the length of each main renal artery, but radially staggered to reduce the risk of intermediate-term renal artery stenosis. SYMPLICITY HTN-1 was the proof-of-principle trial that prompted great enthusiasm in the hypertension and interventional communities. ${ }^{26}$ A total of 50 patients were enrolled with resistant hypertension, defined as $\mathrm{SBP} \geq 160 \mathrm{~mm} \mathrm{Hg}$ and on at least three antihypertensive medications (one of which had to be a diuretic). Screening exclusions were glomerular filtration rate (GFR) $<45 \mathrm{~mL} / \mathrm{min}$, type 1 diabetes, hemodynamically significant valvular disease, and renovascular abnormalities (e.g., renal artery stenosis, dual renal artery). Five enrolled subjects were excluded due to anatomic criteria but were followed for the duration of the trial. Subjects undergoing RFA had up to six lesions in each artery. The primary endpoints were 12-month safety and office blood pressure measurements. Secondary endpoints were effect on NEPI spillover and renal function. Investigators noted mean reductions in office blood pressures of 14/10 $\mathrm{mm} \mathrm{Hg}$ at 1 month and 27/17 mm Hg at 12 months. A small subset of patients underwent ambulatory blood pressure monitoring (ABPM), which demonstrated a more modest reduction in mean SBP of $11 \mathrm{~mm} \mathrm{Hg}$. Another subset of patients had analysis of renal NEPI by the isotope dilution spillover method at baseline and then again at 14 to 30 days after RDN. There was a $47 \%$ mean reduction in NEPI, much less than unpublished preclinical work reporting an $85 \%$ reduction and suggesting incomplete sympathetic nerve interruption. There were no postprocedural renovascular complications or change in GFR. Although the reported results seem dramatic, the lack of controls does not allow definitive conclusions to be drawn. Factors that can explain these results include, but are not limited to, unintended exaggeration of baseline $\mathrm{BP}$ and regression to the mean, Haw thorne effect, improved drug compliance, antihypertensive regimen adjustment, lifestyle modifications, and salt restriction.

The impressive results of this 1st-in-man trial of catheter-based RDN led to the randomized but again unblinded SYMPLICITY HTN-2 trial. ${ }^{27}$ A total of 106 patients with resistant hypertension were enrolled and randomized in a 1:1 fashion to RDN or medical therapy.

Baseline blood pressure was 178/96 $\mathrm{mm} \mathrm{Hg}$, and subjects were on an average of $\geq 5$ agents (95\% angiotensinconverting enzyme inhibitors/angiotensin receptor blocker, $90 \%$ diuretics). The primary endpoint was office blood pressure at 6 months. Secondary endpoints included safety, renovascular complications, and change in ABPM. At 6 months, there was a marked $\mathrm{BP}$ reduction in the RDN group vs the control group (33/11 $\mathrm{mm} \mathrm{Hg}$ ). The control group showed no change from baseline (1/0 mm Hg), unlike what is typically seen in the placebo group of a pharmacologic antihypertensive trial. Ambulatory blood pressure monitoring, available for 20 subjects in the RDN group, showed a mean decrease of $11 / 7 \mathrm{~mm} \mathrm{Hg}$, whereas 25 subjects in the control group did not have any change at the final time point. As with SYMPLICITY HTN-1, this raised questions about the validity of the large reductions in office blood pressure. The absence of a true, blinded control group may have masked placebo or Hawthorne effects. On the safety side, there were no renovascular complications and no change in renal function, assessed by serum creatinine and cystatin C, in the RDN group.

The momentum in the field was buoyed by the dramatic results of the SYMPLICITY HTN-1 and HTN-2 trials. As alluded to earlier, a number of other RDN 
platforms were concomitantly developed and tested in uncontrolled, unblinded phase 1 1st-in-man trials. Initial experience with St. Jude's EnligHTN system, an expandable multielectrode basket catheter, was reported in 46 patients with resistant hypertension. ${ }^{28}$ Unlike the SYMPLICITY trials, this study included paired ABPM for all subjects. At 12 months, baseline blood pressure (176/96 $\mathrm{mm} \mathrm{Hg}$ ) fell by an average of $27 / 11 \mathrm{~mm} \mathrm{Hg}$, with a decrease in ABPM of $10 / 6 \mathrm{~mm} \mathrm{Hg}$. There were no major safety events or decrements in renal function. Similar results were seen with Boston Scientific's Vessix system ${ }^{29}$ and Covidien's OneShot device. ${ }^{30}$

Having learned the importance of sham-controlled device trials from prior experiences, including with transmyocardial laser revascularization, ${ }^{31-33}$ investigators set forth on the pivotal SYMPLICITY HTN-3 trial, a randomized, single-blinded, sham-controlled trial in resistant hypertension. ${ }^{34} \mathrm{~A}$ total of 535 subjects were randomized 2:1 to RDN or sham procedure, with the primary endpoint a change in office BP and secondary endpoint a change in ABPM. At 6-month follow-up, there was no significant between-group difference in the change in $\mathrm{BP}$ (mean reduction $14.13 \mathrm{~mm} \mathrm{Hg}$ RDN group vs 11.74 $\mathrm{mm} \mathrm{Hg}$ sham group; $\mathrm{p}=0.26$ with superiority margin of $5 \mathrm{~mm} \mathrm{Hg}$ ). Both groups experienced a similar, albeit smaller, reduction in ABPM readings ( 7 vs $5 \mathrm{~mm} \mathrm{Hg}$ ), again without a statistically significant between-group difference. While safety of the procedure was again confirmed in this large trial, the disappointing results briefly took the wind out of the sails for catheter RDN.

\section{Lesson learned from SYMPLICITY HTN-3 and Preclinical RDN Anatomic Studies}

Since the publication of SYMPLICITY HTN-3 in early 2014, there has been a great deal of analysis and discussion for why the trial failed to meet its efficacy endpoints.

Possibilities include a substantial sham-procedure placebo effect not present in the uncontrolled and nonsham studies, regression to the mean of the highest pressures at enrollment, a limited role for the SNS in some patients with resistant hypertension, lack of measures for intraprocedural feedback, and suboptimal understanding of neurovascular anatomy. Post hoc analysis of SYMPLICITY HTN-3 explored potential confounders in three areas: Medication changes, subgroup differences, and technical details of procedure performance. ${ }^{35}$ Despite trial protocol mandating otherwise, approximately $40 \%$ of patients underwent medication changes over the 6-month follow-up period. The larger-than-expected blood pressure reduction in the sham-control group suggests possible changes in medication adherence. The procedure and hospitalization for the control group obviously exceeds that for control groups in antihypertensive pharmaceutical trials and may have had a more substantial impact. Unlike the earlier SYMPLICITY trials carried out in Europe and Australia, the pivotal US trial population included one-quarter African-Americans. It is not clear why such patients in the sham-control group showed a much larger reduction in blood pressure compared with non-African-American subjects, but possibilities include changes in medication adherence and the influence of vasodilator therapy, used more commonly in the AfricanAmerican subjects. Renal denervation procedures were analyzed according to number of ablations attempted and whether four-quadrant ablation was delivered to both, one, or neither renal arteries. There was a statistically significant difference in blood pressure with increasing number of ablation to 12 or more. Four-quadrant ablation in both renal arteries, thought to be achieved in only a small minority of RDN patients, was associated with a greater reduction in blood pressure that did not reach statistical significance. The absence of procedural feedback and assumptions made about procedural adequacy using X-ray guidance to achieve circumferential disruption of sympathetic fibers suggested the need for better histopathologic knowledge and a more critical platform assessment. The most likely contributor, however, is incomplete or partial denervation. The lesions placed and the geometric patterns were inadequate. It seems that we need at least close to $90 \%$ renal fiber interruption to get clinical results, of which current studies appear to have fallen far short. Additional preclinical and clinical pathology studies have shed light on aspects of nerve anatomy that should favorably inform ongoing efforts.

Tellez et al ${ }^{36}$ reported their findings on the distribution and density of perirenal nerves in miniature swine. They examined $0.5 \mathrm{~mm}$ layers radially to a depth of $10 \mathrm{~mm}$, more than twice that of earlier studies, and found that more than $50 \%$ were located within $3 \mathrm{~mm}$ of the arterial lumen. While a previous human postmortem study described that the vast majority of fibers were located within a $2 \mathrm{~mm}$ radius of the renal artery lumen, these investigators found sympathetic nerves up to $10 \mathrm{~mm}$ away, with approximately one-quarter at a depth greater than $5 \mathrm{~mm}$. There was a longitudinal pattern of nerves with the greatest density in the proximal compared with mid and distal segments ( $45 \%$ of total count $v s \sim 25-30 \%$ ). Given the genetic and cardiovascular anatomic similarities of the pig to humans, these findings suggest the tissue depth to which effective platforms must reach for complete renal sympathetic disruption.

Virmani's group ${ }^{37}$ described autopsy results in 20 subjects (12 hypertensive, 8 normotensive). They examined 40 arteries in 300 sections from the proximal segment to just beyond the bifurcation. The anatomy was similar in 
hypertensive and normotensive subjects, with circumferential distribution almost twice as high in the ventral compared with the dorsal quadrants. The maximal mean number of nerves was higher in the proximal and mid compared with the distal segments. The mean nerve distance to the renal artery lumen was greatest in the proximal segments and least in the distal segments ( $3.40 \pm$ 0.78 vs $2.60 \pm 0.77 \mathrm{~mm}$ ). The lower nerve density in distal and dorsal segments, as well as increasing radial distance in the proximal segment, has clear implications for whether and how a single-electrode RFA system can accurately and reproducibly interrupt renal sympathetic signals.

Extending these anatomic data to preclinical correlates of RFA lesion delivery, Tzafriri et al ${ }^{38}$ studied the effect of RDN on miniature swine. With untreated swine as controls, they used a custom multielectrode catheter for bilateral RDN in the treatment group, delivering lesion from the ostium to $6 \mathrm{~mm}$ into the renal artery. They verified the findings above, noting nerves closer to the lumen in the distal artery (median $6.3 \mathrm{~mm}$ at the ostium and $2.0 \mathrm{~mm}$ at $6 \mathrm{~mm}$ inside) and more variability in circumferential distribution at the artery ostium. By histopathologic verification of tissue ablation and nerve destruction, only 1 out of 8 treated arteries was associated with a significant reduction in parenchymal NEPI, showing a much larger ablation depth and area encompassing all four radial quadrants. Only one-third of nerves were affected at the ostium despite a large ablated area; however, nearly $100 \%$ of nerves distally were destroyed, likely due to smaller depth at this location. The investigators observed a monotonic dependence of NEPI levels on the fraction of nerves affected and estimated a requirement of $>50 \%$ destruction to achieve a threshold blood pressure effect.

Whether number and location of lesion placement affect the degree, consistency, and durability of response to RDN was addressed by Mahfoud et $\mathrm{al}^{39}$ in a preclinical study with swine. Using the Medtronic Spyral (Santa Rosa, California) multielectrode catheter, they delivered lesions in the main right renal artery and/or distal right renal artery beyond the bifurcation. Three separate groups of animals helped address the impact of number and location of lesions on efficacy, whether combined treatment of main and branch improved efficacy, and whether observed responses were durable to 28 days. Procedure efficacy was determined by measuring renal tissue NEPI levels and cortical axon density in comparison to the untreated left kidney in each animal. Increasing number of RFA lesions in the main renal artery $(4,8$, or 12$)$ did lead to a reduction in NEPI, but the investigators did not find a clear dose-response relationship to NEPI and axon density. Lesion application to the distal or branch renal arteries did, however, result in more substantial and consistent reduction in these parameters, which was also observed at the 28-day time point. Average lesion depth was found to be approximately $3.5 \mathrm{~mm}$. It remains to be seen whether clinical implementation of distal or branch renal artery RFA lesion delivery increases the risk for clinical renal artery stenosis.

Providing additional insights into failure mechanisms for RFA, Booth et $\mathrm{al}^{40}$ invasively monitored mean arterial pressure (MAP), heart rate, and RBF in sheep, then performed bilateral RDN with the SYMPLICITY catheter in the treatment group. Control animals exhibited typical and unfavorable responses to renal nerve stimulation with increased MAP, heart rate, and decreased RBF. This response was abolished immediately post-RDN but returned to baseline levels in animals studied at 5.5 and 11 months. In addition, tyrosine hydroxylase tissue levels, a marker for renal efferent nerves, and tissue NEPI levels also returned to baseline at these later time points, consistent with reinnervation.

\section{Future Directions}

While the number of published studies has tapered since SYMPLICITY HTN-3, there remains significant interest in the field and commitment to incorporate the lessons of preclinical and clinical neuroanatomy, procedural considerations, and trial design into next steps. It seems clear that any successful implementation of catheter-based RDN must take into consideration several principles: (1) Identify the appropriate group of patients, i.e., patients who have treated or untreated hypertension linked to increased sympathetic renal nerve activity. After all, the intended procedure aims to abolish increased signal trafficking through the renal sympathetic fibers, both afferent and efferent. If sympathetic overactivity is not a prominent feature in these patients, then interruption of fibers will not work. (2) Achieve close to complete sympathetic fiber interruption. This is the conclusion from a plethora of preclinical experimental models. Surgical renal nerve resection in these models decreased renal tissue NEPI by as much as $99 \%$. This degree of denervation is difficult to achieve with the devices and platforms currently available. Recent anatomic descriptions indicate that fibers may course in the adventitia as deep as $12 \mathrm{~mm}$ from the lumen. Fortunately, fiber depth is much in the distal renal artery bifurcation and beyond. Thus, targeting lesion delivery distally and into the branches may achieve better and more complete RDN. Alternative approaches that use neurolytic agent applications may be more effective. (3) Safety needs to be maintained with attention to technique, avoidance of arterial dissections, and/or extensive tissue disruption, all the while achieving near-complete sympathetic fiber interruption. Improved knowledge of longitudinal and radial 
nerve distributions from human and animal pathology studies may allow more rational design or modification of systems and targeting of anatomy. Fibers are more numerous in the ostial segment of the renal artery, but they run much deeper, presenting a challenge for RFA devices which typically cause thermal injury only to $4 \mathrm{~mm}$. While a more distal or branch artery location finds nerves a shorter distance from the lumen, some nerves may separate more proximally and the risk of subsequent renal artery stenosis may be higher with lesion delivery in smaller caliber segments. Ablative solutions offer an innovative platform to surmount some of these challenges and effect RDN. Their Peregrine System has the ability to circumferentially interrupt fibers at radial depths more than $10 \mathrm{~mm} .{ }^{41}$ Introduced through a guide catheter, the delivery device has three 30-gauge needles that are actuated and advanced to a depth of $3.5 \mathrm{~mm}$. A small volume $(\sim 0.5 \mathrm{~mL})$ of alcohol, a potent neurolytic, is delivered via the needles into the perivascular and adventitial space, achieving deep and circumferential nerve interruption. Nerve destruction to radial depths of $13 \mathrm{~mm}$ and parenchymal NEPI reductions near 90\% were achieved in swine, with no adverse effects on excretory function. The 1st-in-man experience with 18 subjects verified safety and tolerability with similar preliminary efficacy as with phase I trials of other devices. Whether these impressive results will be corroborated in larger studies remains to be seen.

The lack of immediate feedback or point-of-care assessment of procedural effectiveness also poses significant challenges for catheter-based RDN.

As suggested in the American Society of Hypertension's Scientific Statement on $\mathrm{RDN}^{42}$ the availability of a biomarker (e.g., NEPI spillover) or physiologic parameter (e.g., RBF) that could be measured immediately and serve as a procedural endpoint might greatly enhance procedural consistency and therapeutic reproducibility. This consensus document also recommended an approach to phase II proof-of-concept studies that parallels pharmacologic antihypertensive trial design. In order to definitively isolate and ascertain the blood pressure lowering effect of RDN using a specific platform, a sham-controlled trial of patients with stable but not severe hypertension (SBP $<160 \mathrm{~mm} \mathrm{Hg}$ ), either untreated or off antihypertensive medications, might more efficiently allocate resources and select promising strategies earlier in the development pipeline. A large body of evidence from phase I and II pharmacologic trials demonstrates the short-term safety of withholding standard antihypertensive therapy in this population. The routine use of ABPM would be preferred and confer the additional benefit of smaller trial sample sizes.

\section{CONCLUSION}

An abundance of preclinical and clinical data support a central role for sympathetic overactivity in the initiation and maintenance of hypertension. Before the availability of oral antihypertensive therapy, neurosurgical approaches to sympathectomy proved valuable in patients with malignant hypertension but were limited by marked side effects. As cardiovascular therapy moved toward catheter-based delivery, a convergence of ideas and technologies led to the resurgence of this sympathoinhibitory approach with transvascular radiofrequency energy delivery to effect periarterial and adventitial nerve interruption. The remarkable blood pressure-lowering results of early SYMPLICITY clinical trials with the single-electrode catheter raised hopes and expectations for treatment across a wide spectrum of conditions characterized by sympathetic overactivity, including resistant hypertension, heart failure, and atrial fibrillation. The pivotal and sham-controlled SYMPLICITY HTN-3 study failed to show a difference between treated and control groups. The subsequent period of analysis and more rigorous preclinical experimentation has yielded useful insights into nerve anatomy and distribution, limitations of current platforms and potential modifications to procedural strategies, and perhaps a more consistent approach to trial design, patient selection, and treatment protocols. The future looks bright and hope remains strong for catheter-based RDN to treat resistant hypertension and possibly many other clinical syndromes.

\section{REFERENCES}

1. Bernard C. Lecons sur les Proprietes physiologiques et les alterations pathologiques des liquides de l'organisme. Vol. 2. Paris: Balliere et Fils; 1859. p. 170-171.

2. Bradford JR. The innervation of the renal blood vessels. J Physiol 1889 Jul;10(5):358-407.

3. Liu L, Barajas L. The rat renal nerves during development. Anat Embryol (Berl) 1993 Oct;188(4):345-361.

4. Kottke FJ, Kubicek WG, Visscher MB. The production of arterial hypertension by chronic renal artery-nerve stimulation. Am J Physiol 1945 Nov;145(1):38-47.

5. Webb RL, Brody MJ. Functional identification of the central projections of afferent renal nerves. Clin Exp Hypertens A 1987 Jan;9(Suppl 1):47-57.

6. Malpas SC, Evans RG. Do different levels and patterns of sympathetic activation all provoke renal vasoconstriction? J Auton Nerv Syst 1998 Mar;69(1):72-82.

7. Thorén P. Efferent renal nerve traffic in the spontaneously hypertensive rat. Clin Exp Hypertens A 1987 Jan;9(Suppl 1): 259-279.

8. Campese VM, Kogosov E. Renal afferent denervation prevents hypertension in rats with chronic renal failure. Hypertension 1995 Apr;25(4 Pt 2):878-882.

9. Campese VM, Kogosov E, Koss M. Renal afferent denervation prevents the progression of renal disease in the renal ablation model of chronic renal failure in the rat. Am J Kidney Dis 1995 Nov;26(5):861-865. 
10. O'Hagan KP, Thomas GD, Zambraski EJ. Renal denervation decreases blood pressure in DOCA-treated miniature swine with established hypertension. Am J Hypertens 1990 Jan;3(1):62-64.

11. Katholi RE, Naftilan AJ, Oparil S. Importance of renal sympathetic tone in the development of DOCA-salt hypertension in the rat. Hypertension 1980 May-Jun;2(3):266-273.

12. Huang WC, Fang TC, Cheng JT. Renal denervation prevents and reverses hyperinsulinemia-induced hypertension in rats. Hypertension 1998 Aug;32(2):249-254.

13. Freyberg RH, Peet MM. The effect on the kidney of bilateral splanchnicectomy in patients with hypertension. J Clin Invest 1937 Jan;16(1):49-65.

14. Adson AW, Brown GE. Malignant hypertension: report of case treated by bilateral section of anterior spinal nerve roots from the sixth thoracic to the second lumbar inclusive. JAMA 1934 Apr;102(14):1115-1118.

15. Sen SK. Some observations on decapsulation and denervation of the kidney. Brit J Urol 1936 Dec;8(4):319-328.

16. Page IH, Heuer GJ. The effect of renal denervation on the level of arterial blood pressure and renal function in essential hypertension. J Clin Invest 1935 Jan;14(1):27-30.

17. Smithwick RH, Thompson JE. Splanchnicectomy for essential hypertension; results in 1,266 cases. J Am Med Assoc 1953 Aug;152(16):1501-1504.

18. Peet MM. Results of bilateral supradiaphragmatic splanchnicectomy for arterial hypertension. N Engl J Med 1947 Feb;236(8):270-277.

19. Keith NM, Wagener HP, Barker NW. Some different types of essential hypertension: their course and prognosis. Am J Med Sci 1939 Mar;197(3):332-343.

20. Kjeldsen SE, Schork NJ, Leren P, Eide IK. Arterial plasma norepinephrine correlates to blood pressure in middle-aged men with sustained essential hypertension. Am Heart J 1989 Oct;118(4):775-781.

21. Smith PA, Graham LN, Mackintosh AF, Stoker JB, Mary DA. Relationship between central sympathetic activity and stages of human hypertension. Am J Hypertens 2004 Mar;17(3): 217-222.

22. Schauerte PN, Scherlag BJ, Scherlag MA, Goli S, Jackman W, Lazzara R. Transvenous parasympathetic cardiac nerve stimulation: an approach for stable sinus rate control. J Cardiovasc Electrophysiol 1999 Nov;10(11):1517-1524.

23. Schauerte P, Scherlag BJ, Pitha J, Scherlag MA, Reynolds D, Lazzara R, Jackman WM. Catheter ablation of cardiac autonomic nerves for prevention of vagal atrial fibrillation. Circulation 2000 Nov;102(22):2774-2780.

24. Papademetriou V, Rashidi AA, Tsioufis C, Doumas M. Renal nerve ablation for resistant hypertension: how did we get here, present status, and future directions. Circulation 2014 Apr;129(13):1440-1451.

25. Papademetriou V, Tsioufis C, Doumas M. Renal denervation and Symplicity HTN-3: "Dubium Sapientiae Initium" (Doubt is the beginning of wisdom). Circ Res 2014 Jul;115(2): 211-214.

26. Krum H, Schlaich M, Whitbourn R, Sobotka PA, Sadowski J, Bartus K, Kapelak B, Walton A, Sievert H, Thambar S, et al. Catheter-based renal sympathetic denervation for resistant hypertension: a multicentre safety and proof-of-principle cohort study. Lancet 2009 Apr;373(9671):1275-1281.

27. Symplicity HTN-2 Investigators, Esler MD, Krum H, Sobotka PA, Schlaich MP, Schmieder RE, Bohm M. Renal sympathetic denervation in patients with treatment-resistant hypertension (The Symplicity HTN-2 Trial): a randomised controlled trial. Lancet 2010 Dec;376(9756):1903-1909.

28. Papademetriou V, Tsioufis CP, Sinhal A, Chew DP, Meredith IT, Malaiapan Y, Worthley MI, Worthley SG. Catheter-based renal denervation for resistant hypertension: 12-month results of the EnligHTN I first-in-human study using a multielectrode ablation system. Hypertension 2014 Sep;64(3):565-572.

29. Sievert H, Schofer J, Ormiston J, Hoppe UC, Meredith IT, Walters DL, Azizi M, Diaz-Cartelle J, Cohen-Mazor M. Renal denervation with a percutaneous bipolar radiofrequency balloon catheter in patients with resistant hypertension: 6-month results from the REDUCE-HTN clinical study. EuroIntervention $2015 \mathrm{Feb}$;10(10):1213-1220.

30. Verheye S, Ormiston J, Bergmann MW, Sievert H, Schwindt A, Werner N, Vogel B, Colombo A. Twelve-month results of the rapid renal sympathetic denervation for resistant hypertension using the OneShot ${ }^{\mathrm{TM}}$ ablation system (RAPID) study. EuroIntervention 2015 Feb;10(10):1221-1229.

31. Frazier OH, March RJ, Horvath KA. Transmyocardial revascularization with a carbon dioxide laser in patients with endstage coronary artery disease. N Engl J Med 1999 Sep;341(14): 1021-1028.

32. Allen KB, Dowling RD, Fudge TL, Schoettle GP, Selinger SL, GangaharDM, AngellWW, PetracekMR, ShaarCJ, O'NeillWW. Comparison of transmyocardial revascularization with medical therapy in patients with refractory angina. N Engl J Med 1999 Sep;341(14):1029-1036.

33. Leon MB, Kornowski R, Downey WE, Weisz G, Baim DS, Bonow RO, Hendel RC, Cohen DJ, Gervino E, Laham R, et al. A blinded, randomized, placebo-controlled trial of percutaneous laser myocardial revascularization to improve angina symptoms in patients with severe coronary disease. J Am Coll Cardiol 2005 Nov;46(10):1812-1819.

34. Bhatt DL, Kandzari DE, O'Neill WW, D'Agostino R, Flack JM, Katzen BT, Leon MB, Liu M, Mauri L, Negoita M, et al. A controlled trial of renal denervation for resistant hypertension. N Engl J Med 2014 Apr;370(15):1393-1401.

35. Kandzari DE, Bhatt DL, Brar S, Devireddy CM, Esler M, Fahy M, Flack FM, Katzen BT, Lea J, Lee DP, et al. Predictors of blood pressure response in the SYMPLICITY HTN-3 trial. Eur Heart J 2015 Jan;36(4):219-227.

36. Tellez A, Rousselle S, Palmiere T, Rate WR 4th, Wicks J, Degrange A, Hyon CM, Gongora CA, Hart R, Grundy W, et al. Renal artery nerve distribution and density in the porcine model: biologic implications for the development of radiofrequency ablation therapies. Transl Res 2013 Dec;162(6):381-389.

37. Sakakura K, Ladich E, Cheng Q, Otsuka F, Yahagi K, Fowler DR, Kolodgie FD, Virmani R, Joner M. Anatomic assessment of sympathetic peri-arterial renal nerves in man. J Am Coll Cardiol 2014 Aug;64(7):635-643.

38. Tzafriri AR, Keating JH, Markham PM, Spognardi AM, Stanley JR, Wong G, Zani BG, Highsmith D, O'Fallon P, Fuimaono $\mathrm{K}$, et al. Arterial microanatomy determines the success of energy-based renal denervation in controlling hypertension. Sci Transl Med 2015 Apr;7(285):285ra65.

39. Mahfoud F, Tunev S, Ewen S, Cremers B, Ruwart J, SchulzJander D, Linz D, Davies J, Kandzari DE, Whitbourn R, et al. Impact of lesion placement on efficacy and safety of catheterbased radiofrequency renal denervation. J Am Coll Cardiol 2015 Oct;66(16):1766-1775. 
40. Booth LC, Nishi EE, Yao ST, Ramchandra R, Lambert GW, Schlaich MP, May CN. Reinnervation of renal afferent and efferent nerves at 5.5 and 11 months after catheter-based radiofrequency renal denervation in sheep. Hypertension 2015 Feb;65(2):393-400.

41. Fischell TA, Fischell DR, Ghazarossian VE, Vega F, Ebner A. Next generation renal denervation: chemical "perivascular" renal denervation with alcohol using a novel drug infusion catheter. Cardiovasc Revasc Med 2015 Jun;16(4):221-227.

42. White WB, Galis ZS, Henegar J, Kandzari DE, Victor R, Sica D, Townsend RR, Turner JR, Virmani R, Mauri L. Renal denervation therapy for hypertension: pathways for moving development forward. J Am Soc Hypertens 2015 May;9(5):341-350. 